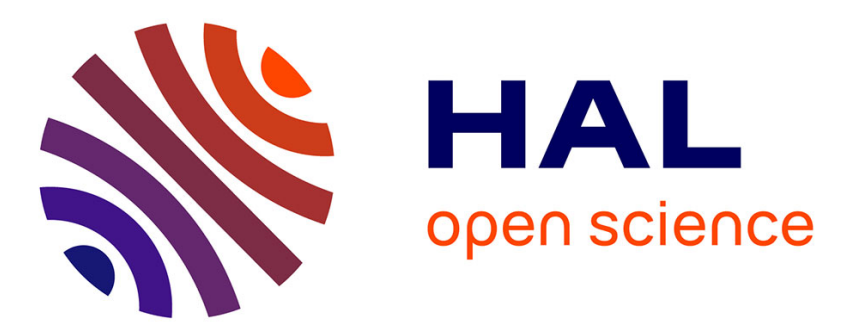

\title{
A Survey of Visible S+ Emission in Io's Plasma Torus during the Hisaki Epoch
}

C. Schmidt, N. Schneider, François Leblanc, C. Gray, J. Morgenthaler, J. Turner, C. Grava

\section{- To cite this version:}

C. Schmidt, N. Schneider, François Leblanc, C. Gray, J. Morgenthaler, et al.. A Survey of Visible S+ Emission in Io's Plasma Torus during the Hisaki Epoch. Journal of Geophysical Research Space Physics, 2018, 123 (7), pp.5610-5624. 10.1029/2018JA025296 . insu-01836498

\section{HAL Id: insu-01836498 https://hal-insu.archives-ouvertes.fr/insu-01836498}

Submitted on 23 Jan 2019

HAL is a multi-disciplinary open access archive for the deposit and dissemination of scientific research documents, whether they are published or not. The documents may come from teaching and research institutions in France or abroad, or from public or private research centers.
L'archive ouverte pluridisciplinaire HAL, est destinée au dépôt et à la diffusion de documents scientifiques de niveau recherche, publiés ou non, émanant des établissements d'enseignement et de recherche français ou étrangers, des laboratoires publics ou privés. 
Key Points:

- New positional information on lo's plasma torus is provided

- Separation of the cold and warm plasma components is modulated by Jovian rotation

- lo enhances the visible plasma torus downstream

Correspondence to:

C. Schmidt,

schmidtc@bu.edu

\section{A Survey of Visible $\mathrm{S}^{+}$Emission in lo's Plasma Torus During the Hisaki Epoch}

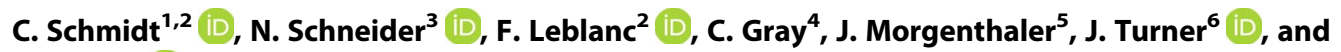 \\ C. Grava' ${ }^{7}$ \\ ${ }^{1}$ Center for Space Physics, Boston University, Boston, MA, USA, ${ }^{2}$ LATMOS/IPSL, UPMC University Paris 06 Sorbonne \\ Universités, UVSQ, CNRS, Paris, France, ${ }^{3}$ Laboratory for Atmospheric and Space Physics, University of Colorado Boulder, \\ Boulder, CO, USA, ${ }^{4}$ Department of Astronomy, New Mexico State University, Las Cruces, NM, USA, ${ }^{5}$ Planetary Science \\ Institute, Tucson, AZ, USA, ${ }^{6}$ Department of Astronomy, University of Virginia, Charlottesville, VA, USA, ${ }^{7}$ Southwest Research \\ Institute, San Antonio, TX, USA
}

Abstract To complement Hisaki's ultraviolet monitoring of the lo plasma torus, a ground-based campaign assembled a comprehensive data set at visible wavelengths with the ARC 3.5-m telescope at Apache Point Observatory. This work concentrates on the bright $\mathrm{S}^{+}$emissions in these data. Recurrent traits in the intensity and location are discerned as a function of Jovian longitude $\left(\lambda_{\text {III }}\right)$. The longitudinal brightness structure differs from past data sets in the visible but is broadly consistent with that of lo's auroral footprint and concurrent Hisaki extreme ultraviolet measurements. Positions of the ribbon feature with $\lambda_{\text {III }}$ confirm that it exhibits a radial wobbling motion that is lesser than the centrifugal limit along a given L-shell. We also find that the radial separation between the cold torus and the ribbon is modulated as Jupiter rotates. The torus is displaced $0.13 R$, dawnward, on average, by an electric field with mean strength $3.8 \mathrm{mV} / \mathrm{m}$, consistent with that inferred by analysis of Hisaki's dawn-dusk brightness asymmetry. The lesser visible brightness asymmetry is uncorrelated to this field strength, however. $\mathrm{S}^{+}$emissions are enhanced downstream of lo, principally at Jovian dawn. lo interacts with the densest region at specific Jovian longitudes and local times; near $\lambda_{\text {III }} \sim 130^{\circ}$ and dawn the ribbon is farthest from Jupiter but still passes radially interior to the satellite. The electron density sweeping past lo and/or its radial proximity to the passing ribbon may also influence brightness, but $\mathrm{S}^{+}$emissions are not governed by a single predominant driver.

\section{Introduction}

At visible wavelengths, ground-based spectroscopy and narrowband imaging can record emissions in all primary species of lo's inner plasma torus: $\mathrm{S}^{+}, \mathrm{S}^{++}$, and $\mathrm{O}^{+}$. These are forbidden electric quadrupole and magnetic dipole transitions, excited through Coulomb collisions with cold thermal electrons, requiring only 1.8 to $3.4 \mathrm{eV}$. The bulk of the energy is radiated in the extreme ultraviolet (EUV) as probed by Voyager, EUV Explorer, Far Ultraviolet Spectroscopic Explorer, Cassini, and currently Hisaki (e.g., Feldman et al., 2004; Herbert et al., 2001; Sandel \& Broadfoot, 1982a; Steffl et al., 2004; Yoshioka et al., 2014). EUV emissions require superthermal electrons $>10 \mathrm{eV}$ and are most sensitive to changes in electron temperature $\left(\propto T_{e^{-}}{ }^{4} ;\right.$ Schneider et al., 1997), whereas visible wavelength emissions are more influenced by colder bulk electron density $\left(\propto n_{e-}\right.$ $n_{i+} \approx n_{e^{-}}{ }^{2}$ ).

The brightness of the torus exhibits distinct radial and latitudinal structure. The most prominent feature is a narrow region, roughly $0.2 R_{\mathrm{J}}$ in width by $1 R_{\mathrm{J}}$ in height, commonly referred to as the ribbon (Trauger, 1984). The ribbon generally dominates torus emissions, illuminating its peak flux tube content (Bagenal, 1994). The ribbon lies just interior to lo's $5.905 R$, radius and occasionally intersects the orbit due to various offsets. It also separates the cold, centrifugally confined inner torus from the warm outer torus, where ion bounce motion along field lines is longer causing the plasma distribution to grow in latitude. Two end-member hypotheses for the ribbons origin have been debated for decades (Thomas et al., 2004). The first hypothesis contends that the ribbon is fresh and hot, formed from the stream of plasma recently created as the torus sweeps by lo. The stream of plasma would be carried downstream from lo, undergoing oscillations about the centrifugal equator until thermalization or multiple passes by lo spread the stream vertically into a ribbon. The second hypothesis suggests that the ribbon is old and cold, formed from plasma created farther out, which slowly diffuses inward against the centrifugal gradient. The slow inward diffusion allows the plasma from the 
warm torus to cool and contract vertically, enhancing the emission to form a distinct feature. In this scenario, continued inward diffusion and cooling forms the washer-shaped cold torus (Herbert et al., 2008). Each hypothesis makes different predictions about the properties of the ribbon-especially its response to recent plasma injection at lo.

A long history of observations have identified $S^{+}$brightness variations that are dependent on Jovian longitude (System III, hereafter $\lambda_{\text {III }}$ Morgan, 1985a; Schneider \& Trauger, 1995; Steffl et al., 2006; Thomas, 1993). Longterm observations are essential to discern $\lambda_{\text {III }}$ structure from the influences independent of Jovian rotation, such as lo or $\lambda_{\mathrm{IV}}$ synchronous drivers (Brown, 1995; Woodward Jr. et al., 1997), their beat frequencies (Steffl et al., 2008), and the stochastic input of neutral cloud sources by volcanism (Koga et al., 2018). Several visible wavelength observers have measured a persistent brightness enhancement near $180^{\circ}$ Jovian longitude-so much so that the proximate region became referred to as the active sector (e.g., Brown, 1995; Pilcher \& Morgan, 1980; Schneider \& Trauger, 1995). A secondary peak near $300^{\circ}$ is less frequently seen (Morgan, 1985a; Pilcher et al., 1985). Although fluctuations in density and parallel plasma temperatures synchronous to Jupiter's rotation are important in shaping these structures (Schneider et al., 1997; Steffl et al., 2006), a review by Thomas et al. (2004) notes that there is little consensus on the cause of the active regions.

The torus also shows EUV brightness enhancements that are synchronous with lo's orbital period. These occur downstream of lo and have been interpreted as local electron heating at, or just downstream of, lo (Sandel \& Broadfoot, 1982b; Tsuchiya et al., 2015). Such structure is noticeably absent in visible wavelengths. While modulation near the 10-hr Jovian rotation period is strong, lo's 42-hr period does not reveal itself in periodograms of visible $S^{+}$emissions (Brown, 1995; Nozawa et al., 2004). This is consistent with Sandel and Broadfoot's (1982b) assertion that lo's passage increases electron temperature, but not electron density, and supports the old and cold perspective on the origins of the ribbon.

The torus has a persistent asymmetry in Jovian local time, which has been almost exclusively observed near the dawn and dusk ansae, points where the emission column appears maximal from Earth's viewpoint. In the EUV, estimates of its dusk/dawn ratio in ansae brightness range between 1.35 (Sandel \& Broadfoot, 1982b), 1.3 (Steffl et al., 2004), and 1.5 (Murakami et al., 2016). Local time displacement of the torus was first postulated to explain such measurements since adiabatic compression close to Jupiter would cause increased collisions there, producing more emission (Barbosa \& Kivelson, 1983; Ip \& Goertz, 1983). Displacement was attributed to the convection electric field set up by the $\mathbf{V} \times \mathbf{B}$ of bulk plasma loss down the Jovian magnetotail and a 4-mV/m symmetric field was inferred. This field's magnitude, variability, and average orientation remain poorly characterized. Subsequently, Dessler and Sandel (1992) and Schneider and Trauger (1995) measured a bulk offset toward dawn of 0.19 and $0.14 R_{\mathrm{J}}$, respectively, confirming the theory. Based on Voyager 2 ultraviolet spectrometer positions, Dessler and Sandel (1992) suggested an $E$ field of $9 \mathrm{mV} / \mathrm{m}$ but concentrated at dusk with and modulated with Jovian longitude. Smyth et al. (2011) determined field strengths ranging $4.1 \mathrm{mV} / \mathrm{m}$ (ground-based $\mathrm{S}^{+}$), $6.4 \mathrm{mV} / \mathrm{m}$ (Galileo J0), and $6.5 \mathrm{mV} / \mathrm{m}$ (Voyager 1). From Galileo and Voyager in situ passes, their model derived an orientation $+20^{\circ}$ from true dawn-dusk in a righthanded convention. However, model fits to the field orientation using ground-based data were nonunique. Murakami et al. (2016) found that a 3.8- $\mathrm{mV} / \mathrm{m}$ field is consistent with Hisaki's 1.5 dawn-dusk brightness ratio and attributed values up to $8.6 \mathrm{mV} / \mathrm{m}$ to forcing by enhanced solar wind ram pressure.

The present work seeks to better characterize the position and brightness of the ribbon through an extensive data set of 244 observations spanning 23 nights over 3.5 years. This analysis focuses on the $\mathrm{S}^{+}$ribbon exclusively, and consideration to the other ion species is deferred to future work. Observational methodology is discussed in section 2. Offsets in the torus' position from electromagnetic forcing are characterized in sections 3.1 and 3.2. Emission brightness as a function of Jovian longitude is described in section 3.3. Section 4 discusses these results in the context of earlier studies, including origins of the observed behavior and potential relationships between position and brightness. Section 5 summarizes key findings.

\section{Observations}

The torus was observed over 23 nights with the dual imaging spectrograph on the Astrophysics Research Consortium 3.5-m telescope at Apache Point Observatory. At a resolving power of $\sim 5,000$, dual imaging spectrograph uses a dichroic to record a spectrum at red and blue wavelengths onto two CCDs (charge coupled 

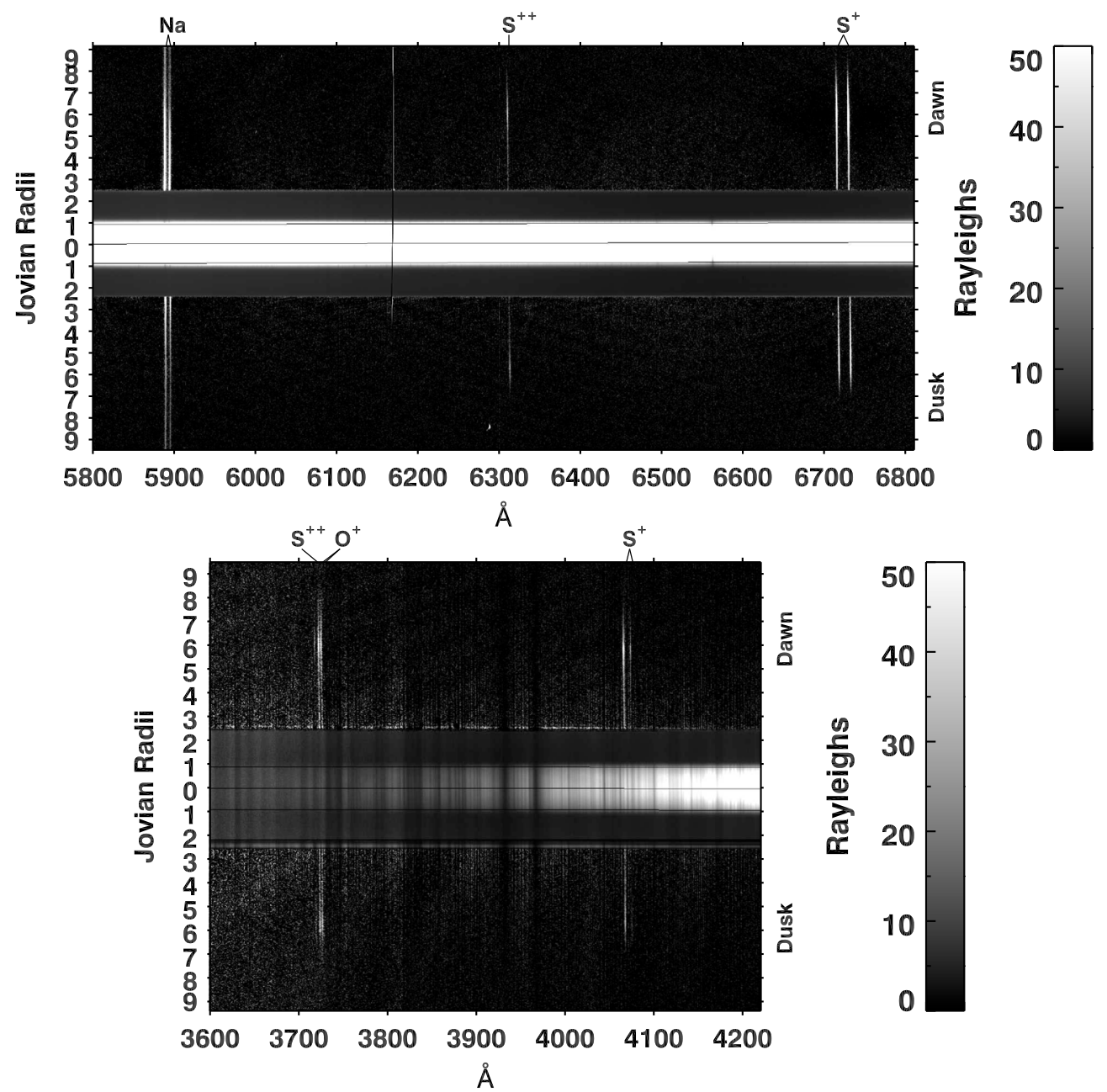

Figure 1. (top) Example frames taken 30 October 2014. Jupiter's emission in the masked region has been reinserted for illustration, as it is zeroed in the subtraction of frames aligned on torus and off torus. Guidelines used for spatial registration show the Jovian centroid and $\pm 0.9 R$ J. The neutral Na cloud is resonantly scattered at 5,890 and 5,896 A. Collisionally excited forbidden transitions are seen from $\mathrm{S}^{++}$at $6,312 \AA$ and from $\mathrm{S}^{+}$at 6,716 and $6,731 \AA$. These are tilted due to the plasma's Doppler shift, though deviations from corotation cannot be constrained at this spectral resolution. $A$ bad pixel column is present at $6,170 \AA$. (bottom) Equivalent for the dual imaging spectrograph blue channel showing collisionally excited transitions from $\mathrm{S}^{++}$at $3,722 \AA, \mathrm{O}^{+}$at 3,726 and $3,729 \AA$, and $\mathrm{S}^{+}$at 4,069 and $4,076 \AA$.

devices). Its slit dimensions are 0.9 arcseconds in width by 6 arcminutes in length, typically extending to $\sim 9$ Jovian radii on both the dawn and dusk sides of Jupiter. The spatial resolution of 0.392 arcseconds per pixel spans more than 100 elements across Jupiter's disk. An antireflective filter is used as a slit mask to reduce Jovian emissions by $\sim 10^{-3}$ (Kodak Wratten 2 gelatin ND3). This mask's spectral density is calibrated using the Jovian reflectance on and off mask, normalizing for the exposure time. The setup allows Jupiter's disk to be measured concurrently with torus emissions. This is useful both for spatial registration and as a standard for absolute brightness calibration. Solar irradiance and Jupiter's albedo are both well known (Karkoschka, 1998; Kurucz, 2005) and the reflectivity of the planet's equatorial zone is relatively stable, varying $\sim 3 \%$ longitudinally (Chanover et al., 1996), with possible temporal evolution up to $5-10 \%$ (Mendikoa et al., 2017).

In order to isolate torus emission from the bright Jovian background, exposures are taken with the slit aligned on and off the centrifugal equator and subtracted. Generally, the observing sequence uses two 10-min integrations with the slit aligned to the centrifugal equator, followed by an exposure with the slit orientation pivoted off torus. At the midexposure time of a torus-aligned observation, the slit's position angle, $\alpha$, is the addition of the Jovian north pole position angle, $\beta_{\mathrm{NP}}$, and the phased tilt of the centrifugal equator: 
Table 1

Spectral Observations of the lo Plasma Torus From Apache Point

\begin{tabular}{lccccc}
\hline Date & Timespan (UT) & $\begin{array}{c}\text { Number of } \\
\text { frames }(244 \text { total) }\end{array}$ & CML & $\begin{array}{c}\text { lo orbital } \\
\text { phase }\end{array}$ & $\begin{array}{c}\text { Jupiter } \\
\text { phase angle }\end{array}$ \\
\hline 7 November 2013 & $11: 37-12: 22$ & 4 & $118-145$ & $120-126$ & -10.0 \\
9 November 2013 & $11: 13-12: 34$ & 5 & $44-94$ & $163-174$ & -9.8 \\
17 December 2013 & $07: 55-10: 22$ & 9 & $250-339$ & $305-326$ & -4.1 \\
24 December 2013 & $07: 58-13: 16$ & 10 & $227-59$ & $290-334$ & -2.7 \\
7 January 2014 & $01: 47-06: 42$ & 15 & $311-130$ & $205-247$ & 0.3 \\
9 February 2014 & $01: 55-02: 41$ & 4 & $247-275$ & $78-85$ & 6.8 \\
14 February 2014 & $01: 29-05: 51$ & 8 & $264-67$ & $11-48$ & 7.6 \\
16 February 2014 & $04: 16-06: 37$ & 4 & $297-38$ & $80-103$ & 7.9 \\
19 February 2014 & $01: 33-06: 42$ & 12 & $299-125$ & $309-352$ & 8.3 \\
30 October 2014 & $08: 35-11: 51$ & 11 & $76-194$ & $350-18$ & -10.5 \\
1 November 2014 & $08: 37-12: 02$ & 12 & $18-141$ & $37-66$ & -10.6 \\
27 March 2015 & $02: 04-05: 31$ & 11 & $170-295$ & $160-190$ & 8.6 \\
6 April 2016 & $02: 31-07: 07$ & 17 & $237-43$ & $327-6$ & 5.6 \\
14 April 2016 & $02: 41-05: 22$ & 12 & $7-104$ & $155-178$ & 6.9 \\
15 April 2016 & $02: 43-04: 26$ & 8 & $159-221$ & $359-13$ & 7.0 \\
10 March 2017 & $07: 52-12: 30$ & 18 & $155-323$ & $6-45$ & -5.4 \\
25 March 2017 & $07: 37-11: 54$ & 19 & $245-41$ & $175-211$ & -2.7 \\
31 March 2017 & $08: 48-12: 03$ & 12 & $113-230$ & $326-353$ & -1.5 \\
11 May 2017 & $02: 44-07: 26$ & 21 & $308-119$ & $334-13$ & 6.3 \\
19 May 2017 & $04: 50-06: 28$ & 8 & $149-208$ & $179-193$ & 7.5 \\
4 July 2017 & $05: 07-06: 03$ & 5 & $241-275$ & $178-186$ & 10.8 \\
7 July 2017 & $03: 12-05: 48$ & 12 & $262-357$ & $51-73$ & 10.8 \\
14 July 2017 & $03: 02-04: 33$ & 7 & $229-284$ & $33-46$ & 10.7 \\
\hline
\end{tabular}

Note. $\mathrm{CML}=$ central meridian longitude.

$\alpha=\beta_{\mathrm{NP}}+6.4 \sin \left(201.7 \times \lambda_{\mathrm{CML}}\right)$ where $\lambda_{\mathrm{CML}}$ is the central meridian longitude of the observer (Acuña et al., 1983; Hill \& Michel, 1976). While Jupiter serves as a spatial reference along the slit, pointing errors up to a few arcseconds may occur in latitude. This does not pose considerable problems because the ribbon's latitudinal extent is roughly 10 times this margin, and brightness of the more centrifugally confined cold torus is not considered in this analysis. Figure 1 shows an average of 10 frames taken UT 30 October 2014 where the background Jovian scattered light has been subtracted.

These 244 spectra, spanning both ansae, comprise the largest spectroscopic data set of the torus assembled in visible wavelengths. Table 1 lists observing times, the number of science frames, the Jovian central meridian longitude toward the observer, lo's orbital phase in the convention where 0 is Jovian midnight, and the phase angle between the Sun, Jupiter, and Earth.

\section{Results}

The core quantity derived from these observations is a profile of a given emission line's photon flux versus distance along the centrifugal equator. Figure 2 gives example profiles on 7 January 2014 for the bright $\mathrm{S}^{+}$ doublet. All Jovian longitudes pass through either ansa in these 15 frames. Fluxes are given in Rayleigh units where $1 R=10^{6}$ photons $\cdot \mathrm{cm}^{-2} \cdot \mathrm{s}^{-1}$ emitting isotopically into $4 \pi$ steradians. Brightness is taken as a sliding average over $0.1 R$, in order to reduce scatter while also keeping within the spatial limitations imposed by atmospheric seeing. The cold torus dominates emissions between 4.5 and $5.2 R_{\mathrm{J}}$, and the more variable ribbon dominates from 5.4 to $6.1 R$. The falloff beyond reflects an increase in sulfur charge states and a decrease in core electron densities in the diffuse warm torus (e.g., Nerney et al., 2017; Yoshioka et al., 2014, 2017). Emission from the ribbon is inseparable from the warm torus over $\lambda_{\text {III }} \sim 210^{\circ}$ to $240^{\circ}$.

To find brightness and radial distance from Jupiter to the ribbon, the $\mathrm{S}^{+}$emission doublet in Figure 2, is summed and fit. An ad hoc functional form that approximates the background between the cold and warm torus is added to a term for the shape of the ribbon feature, which is approximately Gaussian. An overall radial profile of the form 


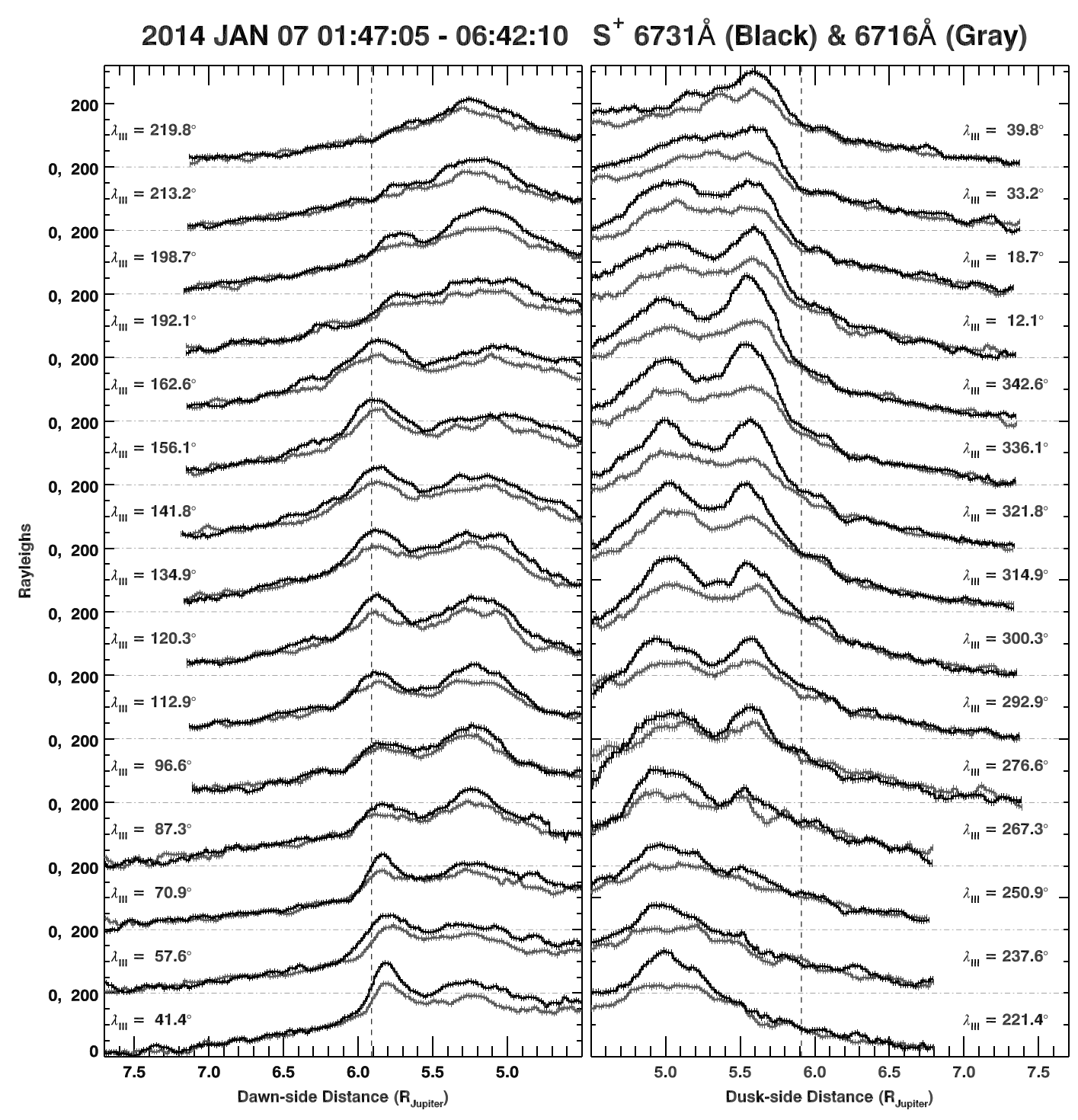

Figure 2. Radial profiles of $S^{+}$doublet emissions on 7 January 2014. A sliding average over $0.1 R$ j is applied. The dashed line shows lo's orbital radius. All Jovian longitudes are sampled in these 15 exposures, but their cadence varies, since isolating torus emission requires the sampling of Jovian scattered light interspersed between science frames. The profiles show little change on 10-min timescales so that hourly morphology is well sampled, such as the ribbon's disappearance near $\lambda_{\text {III }}$ $\sim 225^{\circ}$.

$$
S(r)=A r^{B} \exp \left(|r-C|^{D}\right)+E \exp \left[-\frac{1}{2}\left(\frac{r-F}{G}\right)^{2}\right]
$$

provides reasonable goodness of fit and lower least squares residuals than several other trial functional forms (e.g., quadratic background and Lorenzian ribbon). Here $r$ is the radial coordinate centered on Jupiter and oriented along the centrifugal equator. The variables $A$ through $G$ are fit to the radial profile of the $S^{+}$doublet emissions. The ribbon's emission is described by the Gaussian term and locations of the cold torus and ribbon are approximately given by $C$ and $F$ coefficients, respectively. This approach allows a systematic estimation of the ribbon's brightness and location, taken to be the outer local maxima of equation (1). It also permits the feature's width, $G$, and amplitude, $E$, to be treated as independent parameters.

\subsection{Radial Positions of the Ribbon}

As Jupiter rotates, the tilt and offset of the Jovian magnetosphere give the torus an apparent wobbling motion as viewed from Earth. This apparent motion is mostly latitudinal, but a lesser, radial component is 


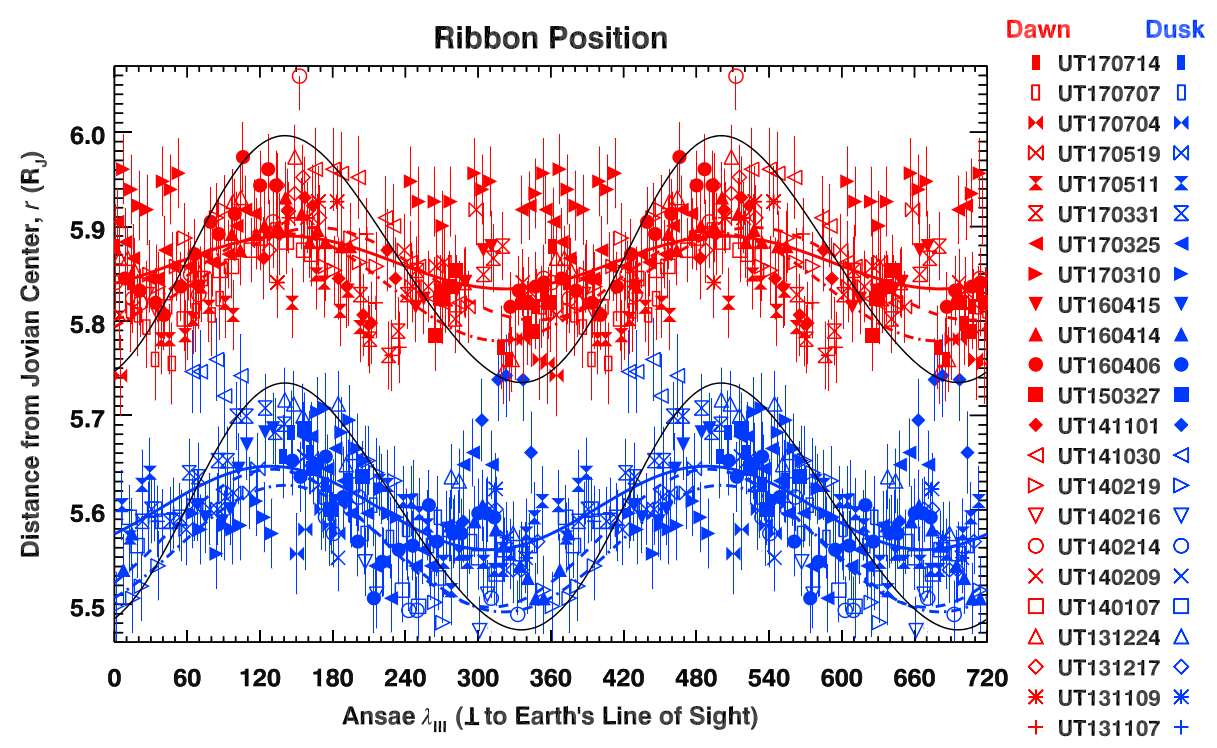

Figure 3. Distance between the ribbon and Jovian center. The dawnside (approaching) ansa is in red and duskside (receding) ansa in blue. Curved lines show best sinusoidal fits (solid) compared to those of Schneider and Trauger (1995; dashed) and Smyth et al. (2011; dotted-dashed). The apparent motion that would be produced by the centrifugal limit along a constant magnetic L-shell is shown in black.

observable in the east-west direction. Figure 3 shows the ribbon's distance from Jupiter along the centrifugal axis. Error bars denote $1 \sigma$ uncertainty in the fit radial position, which added to an assumed systematic error of one pixel. Solid lines show sinusoidal best fits to the present data set; dashed lines denote fits by Schneider and Trauger (1995), and dotted-dashed lines, by Smyth et al. (2011), both using 60 measurements (22 dawn and 38 dusk) on six consecutive nights in 1991. Black lines indicate the centrifugal limit along an arbitrary magnetic $L$-shell (Mcllwain, 1961), fit to an $L$ of 5.87 and $5.61 R_{\mathrm{J}}$ at dawn and dusk, respectively. Each curve represents the distance from Jovian center to the centrifugal point along a dipolar field line at these magnetic radii. Their shape and amplitude are fixed according to an offset tilted dipole model, taken from GSFC O4 (Acuña et al., 1983) and facilitate an unbiased comparison with Smyth et al. (2011). Note that distance from Jupiter's center is plotted here, as opposed to the distance from the spin axis appearing in Schneider and Trauger (1995) and Smyth et al. (2011), though in practice the difference is negligible. Table 2 gives fit parameters and a reduced $\chi^{2}$ goodness-of-fit statistic for each curve in Figure 3.

The mean distance of the dawnside ribbon occurs $0.260 \pm 0.004 R$, farther from Jupiter than it does on the duskside. In $23 \%$ of measurements, the dawnside ribbon appears outside lo's orbit, neglecting the \pm 0.024

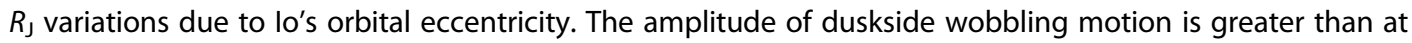
dawn, but neither approaches the centrifugal limit along a given magnetic L-shell as Jupiter rotates $(0.26$ $R$, peak to peak; black curves). Deviations from the nominal wobbling motion are also intrinsic to the torus' behavior; the residual scatter in the data points significantly exceed the error margins regardless of which fit is applied. A small phase lag in $\lambda_{\text {III }}$ modulations is found between dawn and dusk ansae but is not statistically significant ( $14 \pm 9^{\circ}$ difference). The dawnside is nearly phase with motion expected from magnetic geometry, while the duskside phase leads by $2.5 \sigma$.

Table 2

Fit Parameters in the Dawn/Dusk Ribbon Location

\begin{tabular}{lcccc}
\hline & Mean $r\left(R_{\mathrm{J}}\right)$ & Amplitude $H\left(R_{\mathrm{J}}\right)$ & Phase $\varphi\left({ }^{\circ} \lambda_{\text {III }}\right)$ & $\chi_{\mathrm{r}}{ }^{2}$ \\
\hline ST95 Sine & $5.85 / 5.57$ & $0.049 / 0.073$ & $167 / 130$ & $2.29 / 3.33$ \\
SM11 Sine & $5.836 / 5.559$ & $0.057 / 0.067$ & $138 / 141$ & $2.52 / 2.95$ \\
Fit Sine & $5.862 / 5.602$ & $0.028 / 0.044$ & $142 / 128$ & $1.89 / 1.80$ \\
Fit Constant $L$ & $5.863 / 5.601$ & 0.131 (fixed) & 141 (peak; fixed) & $5.71 / 4.64$ \\
\hline
\end{tabular}




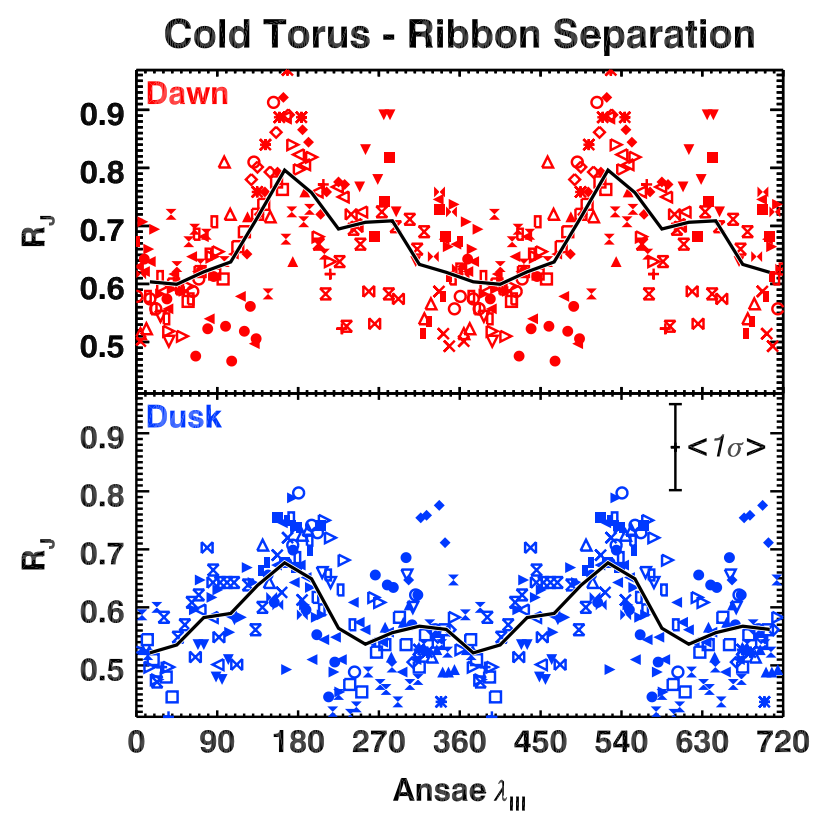

Figure 4. Width of the gap between emission maxima in the ribbon and cold torus, as a function of Jovian longitude. Solid lines are $30^{\circ}$ sliding averages.
As the cold-torus peak is viewed through a column that includes the warm torus and ribbon, its precise radial distance is more difficult to establish. Still, the $\lambda_{\text {III }}$ modulation of its location is distinct from the ribbon and is greater in amplitude. Figure 4 shows the width of the electron trough as a function of $\lambda_{\text {III }}$, which is the separation between emission maxima of the cold torus and the ribbon ( $F-C$ in equation (1)). On both sides of Jupiter, this gap is widest near $170^{\circ}$ and narrowest near $30^{\circ}$. Our finding is associated with the tilt between the cold torus and ribbon planes that Herbert et al. (2008) reported (see their Figure 9). The $1^{\circ}$ to $2^{\circ}$ tilt explanation relates the latitudinal positions of these features, while the relative positions in Figure 4 are radial. Apparent wobbling motion in both these directions is approximately dawn-dusk symmetric. In neither direction is their relative motion convincingly sinusoidal, however, and the crest appears sharper than the trough in Figure 4 . The peak latitudinal separation occurs at $\lambda_{\text {III }} \approx 120^{\circ}$, while the peak in radial separation seen here is at $\lambda_{\text {III }} \approx 170^{\circ}$. The radial separation is single-peaked in $\lambda_{\text {III }}$, whereas a radially symmetric torus would appear double peaked. Altogether, it is evident that a tilt between the cold torus and ribbon planes insufficiently describes the relative position of these two plasma populations. In contrast to the modulation linked to Jupiter's rotation, inspection shows that lo's modulation of the gap width is nearly negligible, although effects very local to the moon cannot be ruled out.

\subsection{The East-West Displacement of the Torus}

The dawn-dusk displacement of the torus is conventionally represented as a distance $\epsilon r$. This displacement results from a dawn-dusk electric field with strength being a fraction, $\epsilon$, of the corotational electric field strength (e.g., Barbosa \& Kivelson, 1983; see their Figure 1) such that

$$
E=-\epsilon \mathbf{V} \times \mathbf{B} \approx \epsilon \frac{2 \pi \Omega r M}{r^{3}}
$$

Here $2 \pi \Omega r$ is the local corotation velocity, the dipole moment, $M$, is 4.278 Gauss $R_{\jmath}{ }^{3}$ (Acuña et al., 1983), and the field falls off as $r^{3}$. Earlier studies, alongside Figure 3, indicate that a slight phase shift may exist whereby the eastern ansa's wobbling motion leads the west (Dessler \& Sandel, 1992; Schneider \& Trauger, 1995). Consequently, if we seek to isolate an electric field that is independent of all forcing tied to Jovian rotation, equation (2) should reflect that a small phase shift may exist between the nominal dawn and dusk wobbling motions. Using the best fit sinusoids from Figure $3, \in$ then becomes

$$
\epsilon=\frac{r_{W}-H_{W} \cos \left(\lambda_{W}-\varphi_{W}\right)-\left[r_{E}-H_{E} \cos \left(\lambda_{E}-\varphi_{E}\right)\right]}{r_{W}-H_{W} \cos \left(\lambda_{W}-\varphi_{W}\right)+\left[r_{E}-H_{E} \cos \left(\lambda_{E}-\varphi_{E}\right)\right]} \approx \frac{r_{W}-r_{E}}{r_{W}+r_{E}}
$$

where $H$ is the amplitude, $\varphi$ is the phase when accounting for this effect, and $E$ and $W$ respectively denote dawn (east) and dusk (west). With this small correction, the convection field is identified as the proportion of the corotational field needed to radially displace both ribbon locations as measured.

Using equations (2) and (3), dawn-dusk convection electric fields are calculated from the 198 dawn-dusk pairs where ribbon features could be detected at both ansae. Figure 5 plots this result with respect to the local time angle from true dawn-dusk, $\Phi$, utilizing the observer's phase angle. Several traits are immediately evident: (1) The $E$ field measurements are highly scattered, (2) each night has a reasonably distinct clumping of $E$, (3) different nights clump to different median $E$ values, and (4) there is no apparent trend of $E$ with $\Phi$. The derived mean field strength from both cold torus and ribbon locations is consistent with $3.8 \mathrm{mV} / \mathrm{m}$-the mean inferred using concurrent brightness asymmetries in Hisaki's spectra (Murakami et al., 2016). A spread of 1 to $7 \mathrm{mV} / \mathrm{m}$ is obtained, while Hisaki's brightness asymmetries suggest that still larger field strengths of up to $8.6 \mathrm{mV} / \mathrm{m}$ may occur sporadically. 


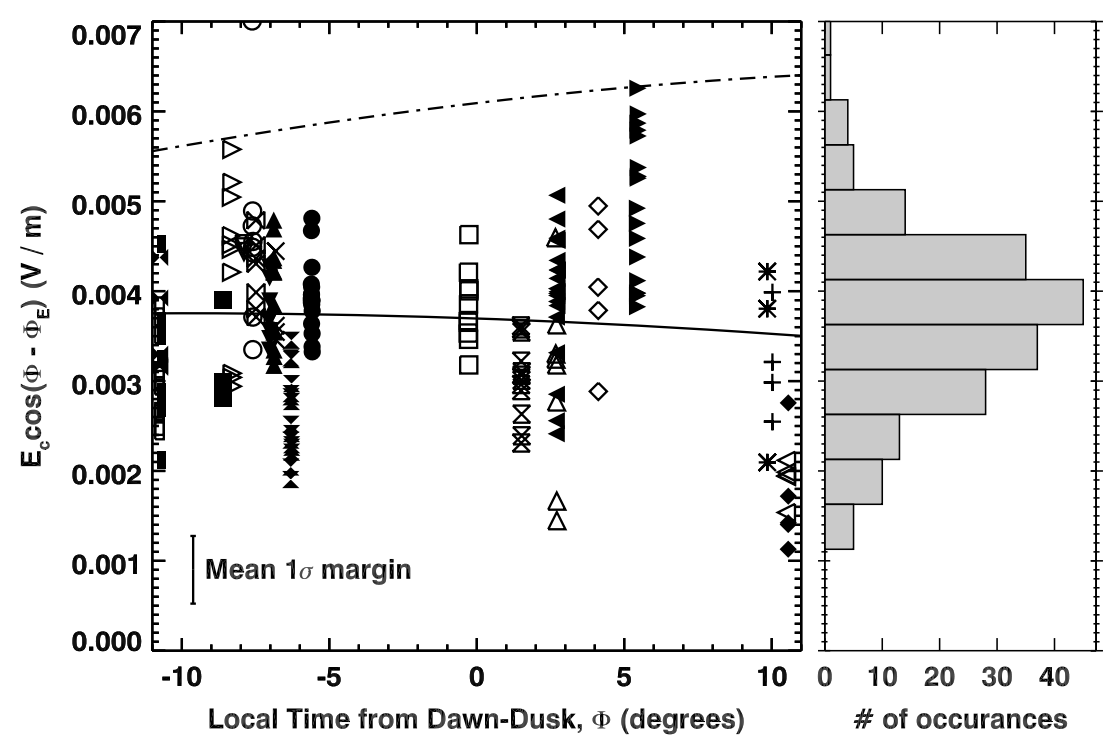

Figure 5. The dawn to dusk convection electric field and its direction derived from the ansae local time as viewed from Earth, that is, parallax. Positive $\Phi$ is clockwise toward a noon-to-midnight direction, and a maxima at $\Phi=0$ would indicate true dawn-to-dusk orientation. The solid line shows best fit $E$ and $\Phi$. The dotted-dashed curve shows these parameters fit by Smyth et al. (2011) to in situ data.

Earth's line-of-sight geometry samples $\pm 11^{\circ}$ in Jovian local time as it orbits the Sun. If the torus' average east-west offset swings slightly within this narrow range, it implies that the electric field deviates from true dawn-dusk orientation. In this way, parallax may help constrain the mean field orientation. Fitting these points suggests an $E$ field of $3.8 \pm 0.1 \mathrm{mV} / \mathrm{m}$ pointing $\Phi_{E}=-10 \pm 4^{\circ}$ clockwise from true dawn-dusk, seen as the solid line of Figure 5. This is inconsistent with the strength and $\Phi_{E}=+20^{\circ}$ orientation that Smyth et al. (2011) proposed from Galileo and Voyager plasma science data (dotted-dashed curve). Constraints on the field orientation herein are imprecise considering the limited geometry that parallax samples and the scatter intrinsic to the torus ( $\sigma=0.9 \mathrm{mV} / \mathrm{m}$ in the histogram at right). Smyth et al. found that fits to the Schneider and Trauger (1995) data set gave nonunique orientations, and the present analysis using parallax cannot improve upon that result, despite a much larger data set. The field strength is clearly lesser than derived from both spacecraft, however, and continued measurement may better constrain orientation using this method.

\subsection{Brightness of the Ribbon}

A suitable metric for the strength of the ribbon feature warrants careful consideration. Figure 6 shows the $\mathrm{S}^{+}$ doublet's brightness as function of $\lambda_{\text {III }}$ and also the integral of the Gaussian term in equation (1), $\sqrt{2 \pi} E G$. While the resulting Rayleighs $R$, units are unconventional in the latter, spatially integrating the ribbon's emission has the benefit that any smearing from pointing errors or seeing effects along the slit aperture are amended through flux conservation. Similar features in $\lambda_{\text {III }}$ envelope are obtained in either case. The longitude structure appears bimodal, though with significant scatter. Maxima near $\lambda_{\text {III }}$ of $120^{\circ}$ and $310^{\circ}$ are far separated from $\lambda_{\text {III }}$ of $22^{\circ}$ and $202^{\circ}$ where the torus appears edge-on, confirming that the opening angle of the torus does not substantially influence the ribbon's flux (Morgan, 1985b; Nozawa, 2001). The apparition of these active regions is only fleeting. Still, the commonality between dawn and dusk extrema is more organized than stochastic variation. Plausible origins for this structure are discussed in section 4.4.

Independent of which brightness metric is used, the overall ratio of dusk to dawn emissions is 1.15-1.2. When comparing this with Hisaki's ratio, it is important to remember that EUV emissions have different electron density and temperature dependence; see section 4.2 for discussion. In a given ground-based observation herein, either side of the torus can be significantly brighter, at times exceeding a factor of 4 with respect to each other. Such extreme brightness asymmetries are higher than any reported previously. These occurrences can be attributed to longitudinal swellings, rather than compression effects from the east-west displacement. Consequently, the brightness ratio alone is not a viable proxy for the electric field on timescales $<5 \mathrm{hr}$. 

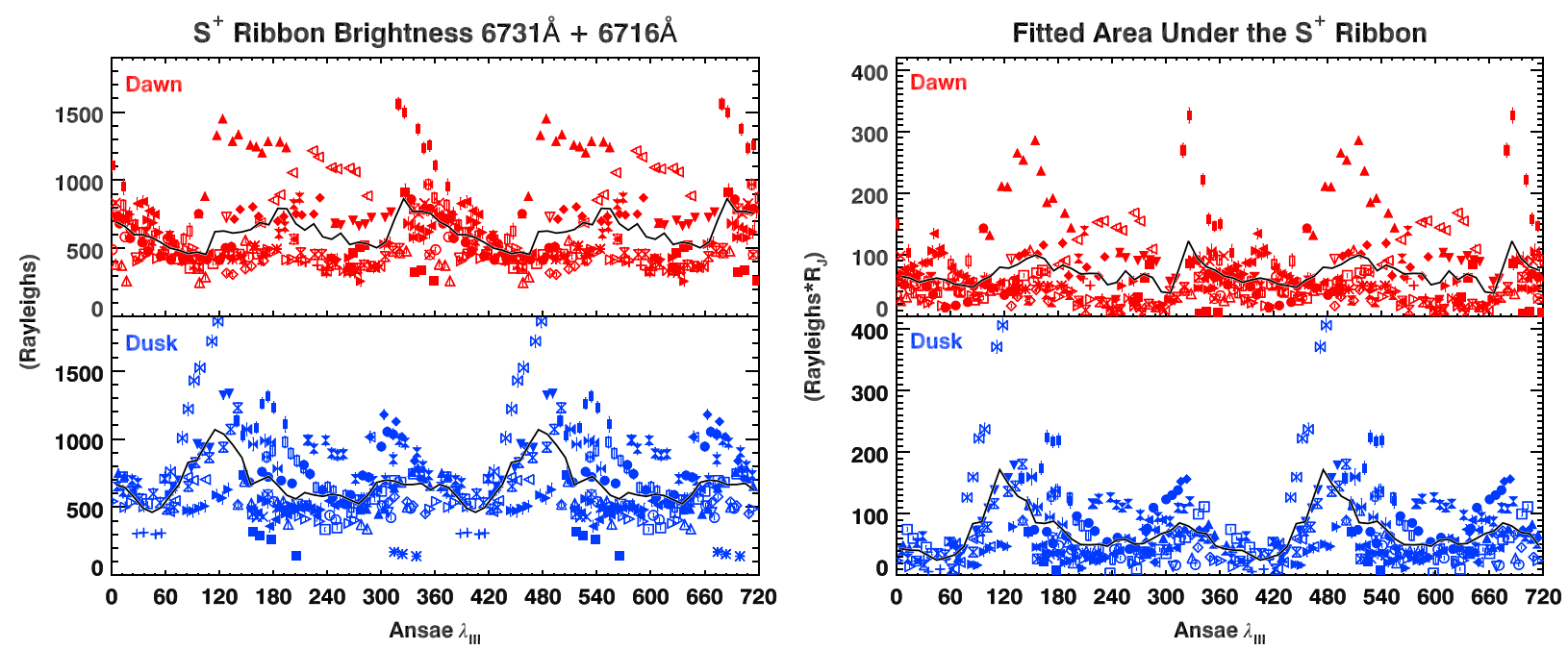

Figure 6. The strength of the ribbon feature in coadded $\mathrm{S}^{+}$emissions versus magnetic longitude. Symbols show different observing dates per Figure 3 . The left panel shows peak brightness, while the right shows the area under the Gaussian fitted in equation (1). The solid line is a sliding average of $10^{\circ}$.

\section{Interpretation, Comparisons, and Discussion}

\subsection{Ribbon Planetocentric Distance and East-West Electric Field}

Observed east-west offsets are lower than several UV and in situ measurements. The mean $\epsilon=0.023$ matches Schneider and Trauger (1995) and Murakami et al. (2016) but differs from the $\epsilon \approx 0.042(6.5 \mathrm{mV} / \mathrm{m})$ inferred from the in situ Voyager 1 plasma science and Galileo data (Smyth et al., 2011) or the $\epsilon \approx 0.033(5.1 \mathrm{mV} / \mathrm{m}$ ) from Voyager 2 ultraviolet spectrometer (Dessler \& Sandel, 1992). These measurements of the offset could be mutually consistent if the torus experienced up to a factor of 2 change in mass convection rates (assuming $E \propto \bigotimes^{3 / 4}$ following Brown \& Bouchez, 1997). Variable convection is not a definitive explanation for discrepancies between observations, however.

Occasional field strengths up to $7 \mathrm{mV} / \mathrm{m}$ are observed, as have been inferred from the EUV dawn-dusk brightness ratio (Murakami et al., 2016). Galileo measurements of dipolarization events in the magnetotail have been used to estimate plasmoid mass loss rates that span more than 2 orders of magnitude (Vogt et al., 2014). The associated $E$ field, however, appears much less variable. This difference may simply reflect that plasmoids pose a small fraction of all plasma loss, as the Galileo analysis concluded, or that the coupling between these two disparate regions is moderated by their distance (Chané et al., 2017). Variation within a given night of observation, as seen Figure 5, is more rapid than timescales expected from plasmoid recurrence. However, as with the cold torus locations, the $E$-field measurement is not immune to line-of-sight effects. A bright ribbon localized over longitudes just interior to the ansa could at times displace the peak from the ansa itself, that is, some radii in Figure 3 may be artificially low. If the $E$ field indeed changes on timescales comparable to a spacecraft's transit time across the torus, in situ particle measurements over a single passage could consequently infer an instantaneous offset substantially different than the mean.

The inferred mean field point toward 17:20 LT or 10 earlier than true dawn-dusk. This differs from prior estimates of 19:00 to 19:20, albeit also with different magnitude (Sandel \& Broadfoot, 1982a; Smyth et al., 2011). However, the field orientation cannot be derived to high precision from the data at hand. Plasmoid events measured by Galileo are most prevalent at 02:00 to 03:00 the postmidnight sector, suggesting a perpendicular $E$ field pointing $\sim 20: 30 \mathrm{hr}$ if these events dominate convection (Vogt et al., 2014). Post dawn-dusk orientation thus supports the idea that the $E$ field results from tailward plasma convection and seems more plausible. Voyager 2 may have in part measured higher amplitude wobble because of its viewing angle, which at $37^{\circ}$, was nearly normal to such an orientation. If the wobble's amplitude scales with the magnetic field strength, Voyager 2's viewing angle was ideal to measure the maximal forcing near duskside, but it remains unclear why a wobble amplitude even larger than magnetic motion was observed (Dessler \& Sandel, 1992). To remain consistent with present data, it is unlikely that the convection $E$ field could sustain an orientation more than $3 \mathrm{hr}$ from true dawn-dusk, a possibility that Cheng et al. (1984) proposed. Were this the case, lo's orbit would 
frequently coincide with the electron trough of plasma depletion (Bagenal, 1994; Herbert et al., 2008). Evacuating iogenic plasma from trough would necessitate rapid, efficient, and complex radial diffusionan implausible scenario.

The east-west apparent wobbling motion of both ansae here are nearly in phase, with the dawnside leading slightly $\left(14 \pm 9^{\circ}\right)$. Were this margin more significant, it would evidence that the torus is out of round. The dawnside led by $37^{\circ}$ in fits by Schneider and Trauger (1995) and Dessler and Sandel (1992) also observed a clear difference in the same direction. Leading dawnside phase thus remains consistent in all measurements of the ribbon's wobbling motion. Given that ribbon's position can deviate from fits in Figure 3 for extended time periods, insufficient data sampling in short-term observations could plausibly indicate high phase shifts. Smyth et al. (2011) attributed the phase difference to sparse data in Schneider and Trauger (1995), but an explanation has not been proposed for this trait in Voyager 2's coverage or in the present measurements at widely separated epochs.

The torus is clearly not a circle centered on Jupiter, since the radial coordinate to both the ribbon and the cold torus fluctuates as Jupiter rotates. Cold plasma guided by the outward centrifugal force of Jupiter's rapid rotation should concentrate farthest from the spin axis along a given field line. Dessler and Sandel (1992) indeed found that the torus' motion approximates an offset tilted dipole's wobble of $0.13 R_{\mathrm{J}}$ at dawn, with an amplitude larger still at dusk. Yet the apparent east-west motions of the ribbon in Schneider and Trauger (1995) are about half this amplitude and in better agreement with the present results. More accurate magnetic models might give centrifugal limits with a better $\chi^{2}$ than the simple constant L-shell seen in Figure 3. However, magnetic geometry and centrifugal forcing cannot match the radial behavior of both the cold torus and ribbon self-consistently, since the two differ substantially in Figure 4. Similarly, magnetic mirror forces and centrifugal forces cannot explain the latitudinal separations between the cold torus and the ribbon (Herbert et al., 2008). A viable mechanism for the relative positions of these plasma populations has yet to be established. Additional clues may be found in their local time differences; the cold torus' radial fluctuation is greater at dawn and available data sets all show that the ribbon's fluctuation with $\lambda_{\text {III }}$ has a larger amplitude on the Jovian duskside.

\subsection{Ribbon Brightness: Local Time Effects}

The dusk ansa is brighter in $60 \%$ of the present measurements and a dusk/dawn ratio of $1.15-1.20$ is consistent with past surveys (Nozawa et al., 2004; Woodward, 1992). In EUV studies, however, such occurs $\geq 90 \%$ of the time and the 1.5 dusk/dawn ratio from Hisaki is significantly higher (Murakami et al., 2016; Steffl et al., 2004). Sensitivity to plasma density at each wavelength is similar, and so this dissimilarity plausibly owes to temperature sensitivity. Standard emission models cannot account for each measured dawn/dusk brightness asymmetry without an associated electron temperature asymmetry. Still, only slight heating is required. For probable conditions in the ribbon $\left(n_{e} \approx 3,000 \mathrm{~cm}^{-3}, T_{e} \approx 4 \mathrm{eV}\right)$, a 1-eV increase in core electron temperature would double the $\mathrm{S}^{++} 680$ - $\AA$ emissivity but have negligible effect on $\mathrm{S}^{+} 6,731$ and 6,716 $\AA$ (CHIANTI 8; Del Zanna et al., 2015). Hisaki analysis has confirmed the earlier reports that core electron temperatures are higher on the Jovian duskside (Hall et al., 1994; Yoshioka et al., 2014), which would indeed yield a higher EUV brightness asymmetry compared to the visible. Unlike the relationship thought to govern the UV, the visible dusk/dawn brightness ratio and electric field strength are uncorrelated $(R=-0.04$; data, $R=-0.08$; fitted ribbon area). Aside from the general condition that the duskside is slightly brighter and closer to Jupiter, the ribbon's brightness does not appear to be governed its planetocentric distance or magnetic L-shell. Correlation coefficients for such relationships are $<|0.2|$. Notably, the brightest measurements are concentrated near their usual planetocentric distances rather than proximal to Jupiter.

\subsection{Ribbon Brightness: Io Phase Effects}

An lo phase effect is well known at EUV wavelengths and generally attributed to plasma heating downstream of the moon (Herbert \& Sandel, 2000; Sandel \& Broadfoot, 1982b; Tsuchiya et al., 2015). Modulation by lo has not been previously observed at visible wavelengths and, in particular, a peak at lo's frequency is absent in all 6,731-Å periodograms showing strong System III and System IV effects (Brown, 1995; Nozawa et al., 2004; Woodward Jr. et al., 1997). However, it is important to note that lo's phase may be poorly sampled in visible wavelengths, since the moon's passage near an instrument's aperture contaminates the spectra. This is not a concern to EUV studies wherein lo's albedo is at most a few percent (Feaga et al., 2004). The narrow 0.9" slit 


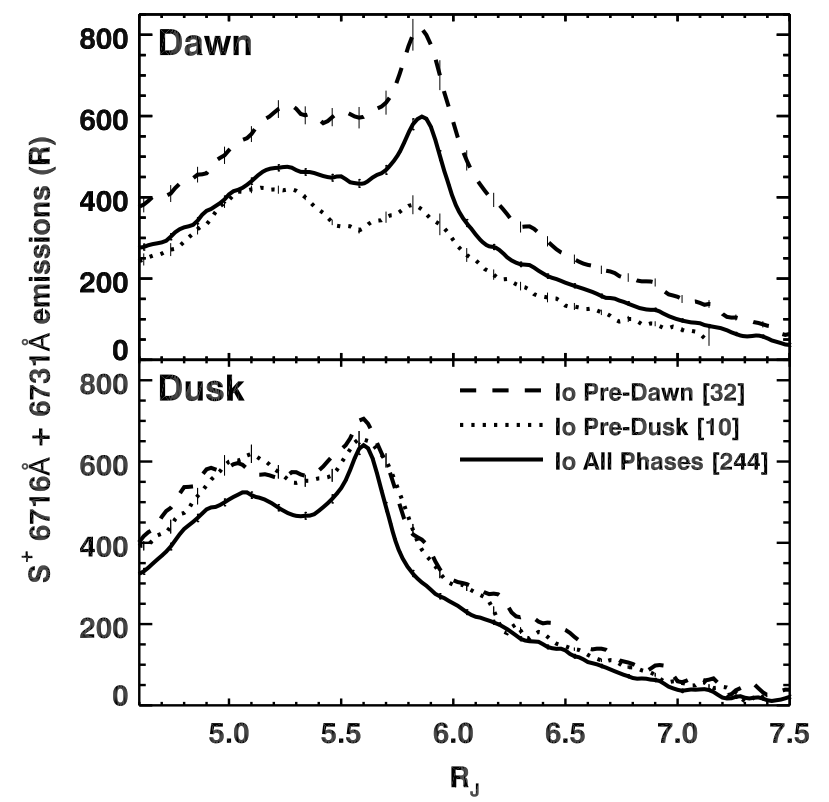

Figure 7. Io's orbital phase effect on $\mathrm{S}^{+}$doublet emissions. Solid lines designate mean emission profiles versus distance from Jupiter, while dashed and dotted lines show only profiles where lo is $<50^{\circ}$ upstream of a dawn or dusk ansae, respectively. Bracketed numbers give the total of frames included.

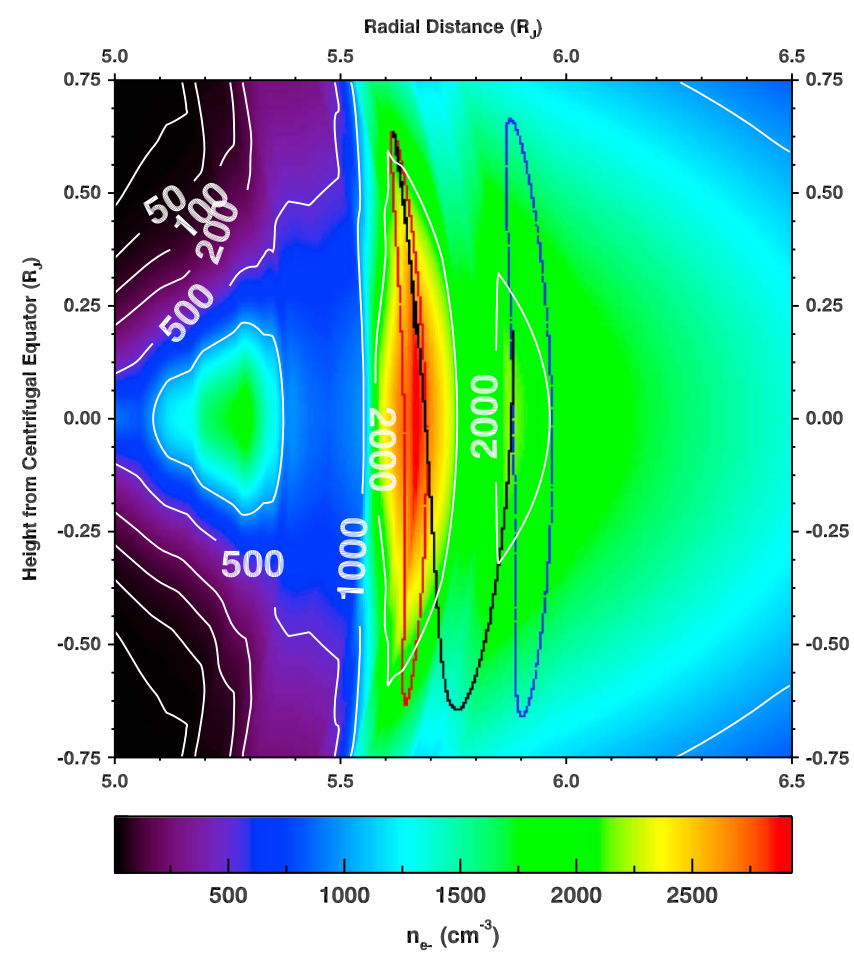

Figure 8. Io's motion with respect to a fixed torus. Electron densities sampled by Voyager 1 plasma science (Bagenal, 1994) are extrapolated in latitude and to a local time of $249^{\circ}$ and $\lambda_{\text {III }}$ of $206^{\circ}$ where the spacecraft encountered the ribbon to offer a snapshot of the torus at a this time. The locations of lo at dawn and dusk local times are shown as red and blue loops, respectively. The black trace shows an example 12.9-hr conjunction period, beginning 15 April 2016 00:00, during which all Jovian longitudes pass lo. makes the present study unique in that it allows for reliable measurements of the visible torus neighboring lo, provided that the two are well separated in latitude, that is, near ansa longitudes $20^{\circ}$ or $200^{\circ}$.

The azimuthal separation between an ansae and lo does seem to affect the ribbon feature and more significantly so on the dawnside of Jupiter. Figure 7 shows the average of all emission profiles, compared to those when either side is $<50^{\circ}$ downstream of lo. The brighter dawnside emission just downstream of lo conceivably results from the dense ribbon just passing very near the moon. Wake enhancements in Hisaki's EUV spectra extend nearly $180^{\circ}$ in azimuth (Tsuchiya et al., 2015). Taking a subset of the visible data in this range, the Spearman correlations between brightness and downstream phase from lo are -0.47 (dawn) and -0.41 (dusk). EUV emissions peak $40-45^{\circ}$ downstream of lo, rather than in the local wake of the moon itself (Sandel \& Broadfoot, 1982b). Herbert and Sandel (2000) attribute this lag to a confluence of enhanced temperature in the immediate wake and enhanced density $\sim 90^{\circ}$ downstream. Available data seem consistent with their theory accounting for emission sensitivity to electron density and temperature. Brightest visible $S^{+}$doublet emissions occurred $\geq 46^{\circ}$ and $\leq 70^{\circ}$ from lo on UT170519 and UT170714, respectively. Hisaki measured a stronger wake enhancement in transitions $<800 \AA$, confirming localized heating (Tsuchiya et al., 2015). Corresponding electrons $>15$ eV can create $\mathrm{S}^{++}$from $\mathrm{S}^{+}$, yet evidently the torus $\mathrm{S}^{+}$ions survive this hot environment and cold $\mathrm{S}^{+}$transitions of just $2 \mathrm{eV}$ are enhanced downstream of the moon.

\subsection{Ribbon Brightness: Jovian Longitude Effects}

Figure 6 shows the apparition of $\lambda_{\text {III }}$ active regions are a transient occurrence within this data set, as several surveys have shown (Bagenal et al., 2016; Steffl et al., 2006), including past 6,731-Å studies (Nozawa et al., 2004). Longitude structure differs from several ground-based studies (e.g., Brown, 1995; Schneider \& Trauger, 1995), but the $\lambda_{\text {III }}$ envelope shows features broadly consistent with concurrent EUV observations (Tsuchiya et al., 2015) and with the $\mathrm{S}^{+}$density and emission in physical chemistry models if simple corotation lag is assumed (Copper et al., 2016). The active region at $120^{\circ}$ leads that described in earlier studies, while the secondary maxima resembles the features previously observed near $\lambda_{\text {III }}$ of $280^{\circ}$ to $300^{\circ}$ (Brown \& Shemansky, 1982; Morgan, 1985a; Pilcher et al., 1985). These significant, albeit transient, brightness enhancements are approximately collocated with the longitudes where the centrifugal and satellite planes intersect near $\lambda_{\text {III }}$ of $110^{\circ}$ and $290^{\circ}$. The structure with $\lambda_{\text {III }}$ in Figure 6 resembles that both in lo's neutral oxygen emission at 6,300 $\AA$ (Oliversen et al., 2001), and in lo's main Alfvén wing auroral footprint (Bonfond et al., 2013; Wannawichian et al., 2013). These traits have been attributed to the variations in the plasma density and magnetic field that lo encounters as its location in the torus changes (e.g., Hess et al., 2013).

Figure 8 shows lo's motion relative to a fixed torus based on Bagenal (1994). The moon's locations at dawn and dusk local times are shown as red and blue loops, respectively, underscoring that a strong interaction with the dense ribbon is unique to Jovian dawn. All Jovian longitudes sweep past lo in $\sim 12.9 \mathrm{hr}$ and an example of such a path on 15 


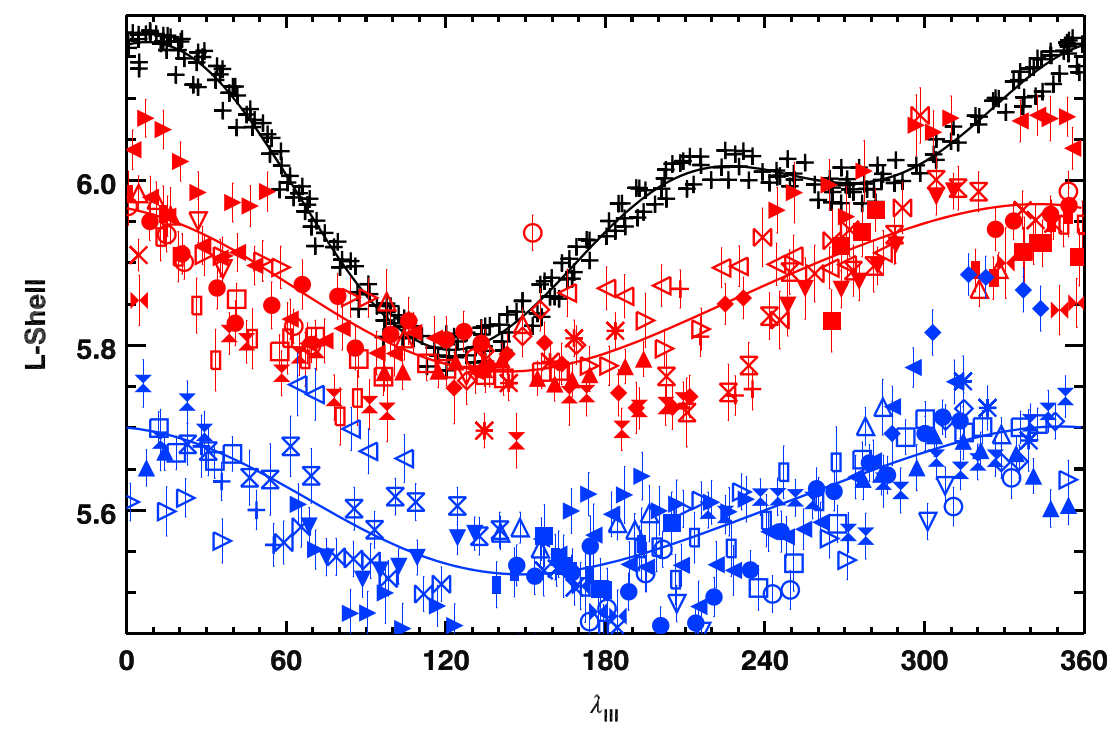

Figure 9. Magnetic L-shells of lo and the ribbon. Symbols follow the same legend as Figure 3 with dawnside measurements in red and duskside in blue. The solid clack curve traces lo's mean orbit of $5.905 R \mathrm{~J}$. Owing to its eccentricity and precession, plus symbols deviate from this slightly and denote lo's L-shell at its last $\lambda_{\text {III }}$ conjunction with the ribbon measurements.

April 2016 is traced in black, covering orbital longitudes from $341^{\circ}$ to Jovian dawn at $90^{\circ}$. During this period, lo crosses the centrifugal equator twice at $\lambda_{\text {III }}$ of $110^{\circ}$ and $290^{\circ}$, as in the emission maxima. The dipole's center is closer to the former, giving this node $10 \%$ greater field strength. Figure 9 shows L-shells of lo and the ribbon to better emphasize this. The proximity of lo's L-shell to the ribbon has a minima at $\lambda_{\text {III }}$ of $17^{\circ}$ and primary and secondary maxima $114^{\circ}$ and $289^{\circ}$, respectively (see also Smyth et al., 2011, Figure 16)-longitudes that approximately mimic the locations of extrema in Figure 6.

Density that lo encounters (Figure 8) and proximity of the ribbon and lo flux tubes (Figure 9) are, of course, related parameters. If either are well correlated with the measured brightness, then it implies the ribbon population is fresh, since corotation lag and System IV slip by $>20^{\circ}$ per day, smearing out discrete $\lambda_{\text {III }}$ structure within a few Jupiter rotations (e.g., Brown, 1995). Both parameters can be estimated by azimuthally interpolating the ribbon coordinates from the observed ansae back to the last azimuthal conjunction with lo and accounting for the ribbon's apparent motion in $\lambda_{\text {III }}$ and local time. Qualitatively, the density sweeping past lo and/or the magnetic separation between lo and the ribbon should modulate the torus in a way that is broadly consistent with the $\lambda_{\text {III }}$ brightness structure in Figure 6 . Quantitatively, we could not confirm that this is the case via statistical correlation. While both plausible drivers offer a bimodel $\lambda_{\text {III }}$ envelope as observed, scatter plots comparing these drivers to individual brightness measurements show correlations are tenuous at best (Pearson's $R=0.27$ ). Tests involving multiple conjunctions with lo, varying the $E$-field orientations and corotation lag did not reveal otherwise. It is noteworthy that the ribbon's magnetic flux tube is nearly always interior that of lo in Figure 9, despite the feature frequently lying radially outside lo's orbit in Figure 3. By interpolating back to their last conjunction, accounting for the ribbon's drift and the moon's orbital precession, it is estimated that the ribbon's flux tube passes interior to lo's field line in $>99 \%$ of all instances.

It is perhaps unsurprising that a potential relationship between brightness and the ribbons radial proximity to lo is difficult to discern, as a combination of multiple influences power emissions. Correlating the ribbon's brightness to its azimuthal separation from lo is also a more straightforward analysis than correlating to its radial separation from lo during its last passage. Furthermore, this estimation of our $n_{e-}$ encountered by lo accounts for its approximate position in the torus but not the plasma scale height or $n_{e-}$ changes that are intrinsic to the local time and $\lambda_{\text {III }}$ coordinates (Herbert et al., 2008; Steffl et al., 2006). The persistent emission minima at $\lambda_{\text {III }} \approx 40^{\circ}$, for instance, may owe to a colocated maxima in the ribbon's parallel ion temperatures that decreases its overall brightness (Schneider et al., 1997). 
Acknowledgments

This work was supported in part by the NASA Solar System Workings Program under SSW16_2-0086 and through CNRS MARMITE (ANR-13-BS05-0012-02). We also value support by the International Space Science Institute (ISSI) in Bern, Switzerland, and the ISSI team "The influence of lo on Jupiter's magnetosphere." Apache Point Observatory staff, especially $R$. McMillan, have aided this project immensely. We thank A. Oza, M. Chaffin S. Rugenski, E. McNeil, K. Connour. L. Bittle, and P. King for their observing support and acknowledge N. Chanover and $\mathrm{A}$. Thelen for coordinating these observations with their own Jovian campaign. The data analyzed in this work are open access http://doi.org/ 10.5281/zenodo.1245697 with IDL analysis code at http://github.com/ carlschmidt.

\section{Conclusions}

An extensive data set of 244 long slit spectra spanning both sides of the lo plasma torus at visible wavelengths is presented. From the brightness and location of $\mathrm{S}^{+}$emissions, key findings can be summarized as follows:

1. Our understanding of the ribbon's apparent radial motion as Jupiter rotates has been refined through these new measurements, but it remains broadly consistent with the earlier characterization. This motion cannot be fully explained by centrifugal forces acting on plasma bound to magnetic field lines of constant potential. Its amplitude is lesser than expected from the centrifugal limit, that is, where the magnetic field lines are farthest from Jupiter's spin axis.

2. The torus extends on average $0.260 R$, farther from Jupiter at dawn than at dusk, confirming predictions from its UV brightness asymmetry in Hisaki's data. The torus' offset is uncorrelated with its visible brightness asymmetry, however. Offsets imply an electric field from bulk plasma convection, which has both short- and long-term variability between 1 and $7 \mathrm{mV} / \mathrm{m}$, a mean strength of $3.8 \mathrm{mV} / \mathrm{m}$, and a standard deviation of $0.9 \mathrm{mV} / \mathrm{m}$. We devised a new technique for measuring the $E$ field's deviation from true dawn-dusk orientation using parallax. While this method is unable to provide conclusive results as applied to the existent data, it may prove effective with continued measurement. Given these substantial changes in field strength, long-term measurements with good sampling over all Jovian phase angles will be key to successfully discerning the $E$ field's mean orientation using this method.

3. The radial extent of the ribbon lies outside lo's orbit in $23 \%$ of observations, distributed over a broad region where longitudes $\sim 135^{\circ}$ pass through dawn Jovian local time. However, in their magnetic coordinates at conjunction, the ribbon feature lies beyond lo in only $1 \%$ of instances, after correcting for local time drift forcing and the moon's precession. From this we deduce that the peak flux tube content of the torus is composed of plasma that has experienced inward radial diffusion.

4. As Jupiter rotates, the cold torus emission feature exhibits radial wobbling motion that is distinct from that of the ribbon. This motion is not sinusoidal, and it shows greater amplitude. This result cannot be attributed to previously proposed deviations from planarity or a tilt between the midplanes of the cold torus and ribbon.

5. Longitudinal variations in the $S^{+}$ribbon's brightness are markedly different from past studies. No active region at longitude $\sim 180^{\circ}$ is observed. Instead, the $\lambda_{\text {III }}$ structure resembles that in the EUV with Hisaki and surveys of lo's auroral footprint with Hubble Space Telescope. Brightness peaks at $\lambda_{\text {III }} \approx 120^{\circ}$ and $300^{\circ}$, near where the orbital and centrifugal planes intersect.

6. The well-known brightness asymmetry between dawn and dusk ansae is a lesser $\sim 15 \%$ at visible $S^{+}$wavelengths. The nominal 30-50\% duskside enhancement in the UV thus cannot be attributed to higher density alone since the visible emissions are more density sensitive. To account for this difference at both wavelengths self-consistently then implies that plasma must be adiabatically heated in the Jovian dusk sector.

7. For the first time in visible observations, lo's phase seem to be influential-predominantly on the Jovian dawnside where the moon interacts with the dense ribbon feature. Additional brightness modulation may result from conditions at lo, such as its radial separation from the ribbon or the regional plasma density, but confirmation by statistical correlation is problematic. Altogether, evidence suggests that a confluence of these effects controls the ribbon emissions observed at each ansae.

Scope of this analysis is restricted to the radial and azimuthal characteristics of bright $\mathrm{S}^{+}$transitions. In future work, other emission lines could be used to constrain plasma parameters and ion mixing ratios, particularly in conjunction with Hisaki where measurements overlap.

\section{References}

Acuña, M. H., Behannon, K. W., \& Connerney, J. E. P. (1983). Jupiter's magnetic field and magnetosphere. In A. J. Dessler (Eds.), Physics of the Jovian Magnetosphere 1-50. Cambridge University Press. https://doi.org/10.1017/CBO9780511564574.003

Bagenal, F. (1994). Empirical model of the lo plasma torus: Voyager measurements. Journal of Geophysical Research, 99(A6), 11,043-11,062. https://doi.org/10.1029/93JA02908

Bagenal, F., Wilson, R. J., Siler, S., Paterson, W. R., \& Kurth, W. S. (2016). Survey of Galileo plasma observations in Jupiter's plasma sheet. Journal of Geophysical Research: Planets, 121, 871-894. https://doi.org/10.1002/2016JE005009

Barbosa, D. D., \& Kivelson, M. G. (1983). Dawn-dusk electric field asymmetry of the lo plasma torus. Geophysical Research Letters, 10(3), 210-213. https://doi.org/10.1029/GL010i003p00210 
Bonfond, B., Hess, S., Gérard, J.-C., Grodent, D., Radioti, A., Chantry, V., et al. (2013). Evolution of the lo footprint brightness I: Far-UV observations. Planetary and Space Science, 88, 64-75.

Brown, M. E. (1995). Periodicities in the lo plasma torus. Journal of Geophysical Research, 100(A11), 21,683-21,695. https://doi.org/10.1029/ 95JA01988

Brown, M. E., \& Bouchez, A. H. (1997). The response of Jupiter's magnetosphere to an outburst on lo. Science, 278(5336), 268-271. https://doi. org/10.1126/science.278.5336.268

Brown, R. A., \& Shemansky, D. E. (1982). On the nature of S II emission from Jupiter's hot plasma torus. The Astrophysical Journal, $263,433-442$. https://doi.org/10.1086/160515

Chané, E., Saur, J., Keppens, R., \& Poedts, S. (2017). How is the Jovian main auroral emission affected by the solar wind? Journal of Geophysical Research: Space Physics, 122, 1960-1978.

Chanover, N. J., Kuehn, D. M., Banfield, D., Momary, T., Beebe, R. F., Baines, K. H., et al. (1996). Absolute reflectivity spectra of Jupiter: $0.25-3.5$ micrometers. Icarus, 121(2), 351-360. https://doi.org/10.1006/icar.1996.0093

Cheng, A. F., Paonessa, M. T., Maclennan, C. G., Lanzerotti, L. J., \& Armstrong, T. P. (1984). Longitudinal asymmetry in the lo plasma torus. Journal of Geophysical Research, 89(A5), 3005-3010. https://doi.org/10.1029/JA089iA05p03005

Copper, M., Delamere, P. A., \& Overcast-Howe, K. (2016). Modeling physical chemistry of the lo plasma torus in two dimensions. Journal of Geophysical Research: Space Physics, 121, 6602-6619. https://doi.org/10.1002/2016JA022767

Del Zanna, G., Dere, K. P., Young, P. R., Landi, E., \& Mason, H. E. (2015). CHIANTI-An atomic database for emission lines. Version 8. Astronomy and Astrophysics, 582, A56. https://doi.org/10.1051/0004-6361/201526827

Dessler, A. J., \& Sandel, B. R. (1992). System III variations in apparent distance of lo plasma torus from Jupiter. Geophysical Research Letters, 19(20), 2099-2102. https://doi.org/10.1029/92GL02380

Feaga, L. M., McGrath, M. A., Feldman, P. D., \& Strobel, D. F. (2004). Detection of atomic chlorine in lo's atmosphere with the Hubble Space Telescope GHRS. The Astrophysical Journal, 610(2), 1191-1198. https://doi.org/10.1086/421862

Feldman, P. D., Strobel, D. F., Moos, H. W., \& Weaver, H. A. (2004). The Far-Ultraviolet Spectrum of the lo Plasma Torus. The Astrophysical Journal, 601(1), 583-591.

Hall, D. T., Bednar, C. J., Durrance, S. T., Feldman, P. D., McGrath, M. A., Moos, H. W., \& Strobel, D. F. (1994). Hopkins Ultraviolet Telescope determination of the lo torus electron temperature. Astrophysical Journal, 420, L45-L48.

Herbert, F., Gladstone, R. G., \& Ballester, G. E. (2001). Extreme Ultraviolet Explorer spectra of the lo plasma torus: Improved spectral resolution and new results. Journal of Geophysical Research, 106(A11), 26,293-26,309. https://doi.org/10.1029/2000JA002501

Herbert, F., \& Sandel, B. R. (2000). Azimuthal variation of ion density and electron temperature in the lo plasma torus. Journal of Geophysical Research, 105, 16,035-16,052.

Herbert, F., Schneider, N. M., \& Dessler, A. J. (2008). New description of lo's cold plasma torus. Journal of Geophysical Research, 113 , A01208. https://doi.org/10.1029/2007JA012555

Hess, S. L. G., Bonfond, B., Chantry, V., Gérard, J.-C., Grodent, D., Jacobsen, S., \& Radioti, A. (2013). Evolution of the lo footprint brightness II: Modeling. Planetary and Space Science, $88,76-85$.

Hill, T. W., \& Michel, F. C. (1976). Heavy ions from the Galilean satellites and the centrifugal distortion of the Jovian magnetosphere. Journal of Geophysical Research, 81, 4561-4565.

Ip, W.-H., \& Goertz, C. K. (1983). An interpretation of the dawn-dusk asymmetry of UV emission from the lo plasma torus. Nature, 302(5905), 232-233. https://doi.org/10.1038/302232a0

Karkoschka, E. (1998). Methane, ammonia, and temperature measurements of the Jovian planets and Titan from CCD-spectrophotometry. Icarus, 133(1), 134-146. https://doi.org/10.1006/icar.1998.5913

Koga, R., Tsuchiya, F., Kagitani, M., Sakanoi, T., Yoneda, M., Yoshioka, K., et al. (2018). The time variation of atomic oxygen emission around lo during a volcanic event observed with Hisaki/EXCEED. Icarus, 299, 300-307. https://doi.org/10.1016/j.icarus.2017.07.024

Kurucz, R. L. (2005). New atlases for solar flux, irradiance, central intensity, and limb intensity. Memorie della Società Astronomica Italiana Supplement, 8, 189.

Mcllwain, C. E. (1961). Coordinates for mapping the distribution of magnetically trapped particles. Journal of Geophysical Research, 66(11), 3681-3691. https://doi.org/10.1029/JZ066i011p03681

Mendikoa, I., Sánchez-Lavega, A., Pérez-Hoyos, S., Hueso, R., Rojas, J. F., \& López-Santiago, J. (2017). Temporal and spatial variations of the absolute reflectivity of Jupiter and Saturn from 0.38 to $1.7 \mu \mathrm{m}$ with PlanetCam-UPV/EHU. Astronomy \& Astrophysics, 607(A72), 13. https:// doi.org/10.1051/0004-6361/201731109

Morgan, J. S. (1985a). Models of the lo torus. Icarus, 63(2), 243-265. https://doi.org/10.1016/0019-1035(85)90009-0

Morgan, J. S. (1985b). Temporal and spatial variations in the lo torus. Icarus, 62(3), 389-414. https://doi.org/10.1016/0019-1035(85)90183-6

Murakami, G., Yoshioka, K., Yamazaki, A., Tsuchiya, F., Kimura, T., Tao, C., et al. (2016). Response of Jupiter's inner magnetosphere to the solar wind derived from extreme ultraviolet monitoring of the lo plasma torus. Geophysical Research Letters, 43, 12,308-12,316. https://doi.org/ 10.1002/2016GL071675

Nerney, E. G., Bagenal, F., \& Steffl, A. J. (2017). lo plasma torus ion composition: Voyager, Galileo, and Cassini. Journal of Geophysical Research: Space Physics, 122, 727-744. https://doi.org/10.1002/2016JA023306

Nozawa, H. (2001). Study on the variability of the Jovian lo plasma torus using ground-based observations of sulfur ion emissions (PhD thesis). Sendai, Japan: Tohoku University.

Nozawa, H., Misawa, H., Takahashi, S., Morioka, A., Okano, S., \& Sood, R. (2004). Long-term variability of [SII] emissions from the lo plasma torus between 1997 and 2000. Journal of Geophysical Research, 109, A07209. https://doi.org/10.1029/2003JA010241

Oliversen, R. J., Scherb, F., Smyth, W. H., Freed, M. E., Woodward, R. C. J., Marconi, M. L., et al. (2001). Sunlit lo atmospheric [OI] 6300Å; emission and the plasma torus. Journal of Geophysical Research, 106(A11), 26,183-26,193. https://doi.org/10.1029/2000JA002507

Pilcher, C. B., Fertel, J. H., \& Morgan, J. S. (1985). Forbidden S II images of the lo torus. The Astrophysical Journal, 291, 377-393. https://doi.org/ $10.1086 / 163076$

Pilcher, C. B., \& Morgan, J. S. (1980). The distribution of S II forbidden line emission around Jupiter. The Astrophysical Journal, 238, 375-380. https://doi.org/10.1086/157994

Sandel, B. R., \& Broadfoot, A. L. (1982a). Discovery of an lo-correlated energy source for lo's hot plasma torus. Journal of Geophysical Research, 87(A4), 2231-2240. https://doi.org/10.1029/JA087iA04p02231

Sandel, B. R., \& Broadfoot, A. L. (1982b). lo's hot plasma torus-A synoptic view from Voyager. Journal of Geophysical Research, 87(A1), 212-218. https://doi.org/10.1029/JA087iA01 p00212

Schneider, N. M., Taylor, M. H., Crary, F. J., \& Trauger, J. T. (1997). On the nature of the $\lambda_{\| 1}$ brightness asymmetry in the lo torus. Journal of Geophysical Research, 102(A9), 19,823-19,833. https://doi.org/10.1029/97JA00773 
Schneider, N. M., \& Trauger, J. T. (1995). The structure of the lo torus. The Astrophysical Journal, 450, 450.

Smyth, W. H., Peterson, C. A., \& Marconi, M. L. (2011). A consistent understanding of the ribbon structure for the lo plasma torus at the Voyager 1, 1991 ground-based, and Galileo J0 epochs. Journal of Geophysical Research, 116, A07205. https://doi.org/10.1029/ 2010JA016094

Steffl, A. J., Delamere, P. A., \& Bagenal, F. (2006). Cassini UVIS observations of the lo plasma torus. III. Observations of temporal and azimuthal variability. Icarus, 180(1), 124-140. https://doi.org/10.1016/j.icarus.2005.07.013

Steffl, A. J., Delamere, P. A., \& Bagenal, F. (2008). Cassini UVIS observations of the lo plasma torus. IV. Modeling temporal and azimuthal variability. Icarus, 194, 153-165.

Steffl, A. J., Stewart, A. I. F., \& Bagenal, F. (2004). Cassini UVIS observations of the lo plasma torus. I. Initial results. Icarus, 172(1), 78-90. https:// doi.org/10.1016/j.icarus.2003.12.027

Thomas, N. (1993). The variability of the lo plasma torus. Journal of Geophysical Research, 98, 18

Thomas, N., Bagenal, F., Hill, T. W., \& Wilson, J. K. (2004). The lo neutral clouds and plasma torus. Jupiter. The planet. Satellites and Magnetosphere, 1, 561-591.

Trauger, J. T. (1984). The Jovian nebula - A post-Voyager perspective. Science, 226, 337-341.

Tsuchiya, F., Kagitani, M., Yoshioka, K., Kimura, T., Murakami, G., Yamazaki, A., et al. (2015). Local electron heating in the lo plasma torus associated with lo from HISAKI satellite observation. Journal of Geophysical Research: Space Physics, 120, 10,317-10,333. https://doi.org/ 10.1002/2015JA021420

Vogt, M. F., Jackman, C. M., Slavin, J. A., Bunce, E. J., Cowley, S. W. H., Kivelson, M. G., \& Khurana, K. K. (2014). Structure and statistical properties of plasmoids in Jupiter's magnetotail. Journal of Geophysical Research: Space Physics, 119, 821-843. https://doi.org/10.1002/2013JA019393

Wannawichian, S., Clarke, J. T., Bagenal, F., Smyth, W. H., Peterson, C. A., \& Nichols, J. D. (2013). Longitudinal modulation of the brightness of Io's auroral footprint emission: Comparison with models. Journal of Geophysical Research: Space Physics, 118, 3336-3345. https://doi.org/ 10.1002/jgra.50346

Woodward, R. C. Jr. (1992). The sulfur emission and periodicity of the Jupiter plasma torus in 1988 (PhD Thesis). Madison: Wisconsin University.

Woodward, R. C. Jr., Scherb, F., \& Roesler, F. L. (1997). Variations in Optical S ${ }^{+}$emission from the lo plasma torus: Evidence for quasi periodicity. The Astrophysical Journal, 479(2), 984-991. https://doi.org/10.1086/303925

Yoshioka, K., Murakami, G., Yamazaki, A., Tsuchiya, F., Kimura, T., Kagitani, M., et al. (2014). Evidence for global electron transportation into the jovian inner magnetosphere. Science, 345(6204), 1581-1584. https://doi.org/10.1126/science.1256259

Yoshioka, K., Tsuchiya, F., Kimura, T., Kagitani, M., Murakami, G., Yamazaki, A., et al. (2017). Radial variation of sulfur and oxygen ions in the lo plasma torus as deduced from remote observations by Hisaki. Journal of Geophysical Research: Space Physics, 122, 2999-3012. https://doi. org/10.1002/2016JA023691 